\title{
Identification of MFGE8 in mesenchymal stem cell secretome as an anti-fibrotic factor in liver fibrosis
}

\author{
Yu Jin Jang, Su Yeon An \& Jong-Hoon Kim* \\ Laboratory of Stem Cells and Tissue Regeneration, Department of Biotechnology, College of Life Sciences \& Biotechnology, Korea \\ University, Seoul 02841, Korea
}

The beneficial paracrine roles of mesenchymal stem cells (MSCs) in tissue repair have potential in therapeutic strategies against various diseases. However, the key therapeutic factors secreted from MSCs and their exact molecular mechanisms of action remain unclear. In this study, the cell-free secretome of umbilical cord-derived MSCs showed significant anti-fibrotic activity in the mouse models of liver fibrosis. The involved action mechanism was the regulation of hepatic stellate cell activation by direct inhibition of the TGF $\beta / S m a d-$ signaling. Antagonizing the milk fat globule-EGF factor 8 (MFGE8) activity blocked the anti-fibrotic effects of the MSC secretome in vitro and in vivo. Moreover, MFGE8 was secreted by MSCs from the umbilical cord as well as other tissues, including teeth and bone marrow. Administration of recombinant MFGE8 protein alone had a significant anti-fibrotic effect in two different models of liver fibrosis. Additionally, MFGE8 downregulated TGF $\beta$ type I receptor expression by binding to $\alpha v \beta 3$ integrin on HSCs. These findings revealed the potential role of MFGE8 in modulating TGF $\beta$-signaling. Thus, MFGE8 could serve as a novel therapeutic agent for liver fibrosis. [BMB Reports 2017; 50(2): 58-59]

Tissue regeneration and repair necessitate compensatory restitution of lost tissues and reorganization of the extracellular matrix (ECM) framework. Many previous studies have shown

*Corresponding author. E-mail: jhkim@korea.ac.kr

https://doi.org/10.5483/BMBRep.2017.50.2.012

Received 21 January 2017

Keywords: Liver fibrosis, MFGE8, MSC, Secretome

Abbreviations: ECM, Extracellular matrix; HSCs, Hepatic stellate cells; MFGE8, Milk fat globule-EGF factor 8; MSCs, Mesenchymal stem cells; TGF $\beta$, Transforming growth factor beta; UCMSCs, Umbilical cord-derived MSCs

Perspective to: Su Yeon An, Yu Jin Jang et al. (2016), Milk Fat Globule-EGF Factor 8, Secreted by Mesenchymal Stem Cells, Protects Against Liver Fibrosis in Mice, Gastroenterology, In press Epub 9 Dec 2016, doi:10.1053/j.gastro.2016.12.003 that tissue reconstruction by grafts of stem cells or their progeny promotes functional recovery of injured tissues, including the liver. MSCs are promising candidates for cell replacement therapy due to their ability to differentiate into osteoblasts, chondrocytes, and adipocytes. Moreover, they may differentiate into broader lineages of cell types such as neurons, pancreatic cells, and hepatic cells, although this ability is still under debate. Recent accumulating evidence shows that MSCs secrete a wide spectrum of soluble factors and may promote host tissue regeneration by paracrine actions rather than differentiation into functional somatic cells. However, the therapeutic agents released by MSCs and their exact mode of action are largely unknown.

Liver fibrosis is one of the major medical problems with significant morbidity and mortality worldwide. Liver fibrosis is caused by excess deposition of ECM in tissues and can develop into cirrhosis or liver cancer. In the early stages of hepatic fibrosis, macrophages are recruited into the liver by chemokines produced from both damaged hepatocytes and endothelial cells and produce various inflammatory cytokines, including TGF $\beta 1$. TGF $\beta$ is the most potent stimulating factor for procollagen I \& III gene transcription. TGF $\beta /$ Smad signaling activates quiescent HSCs into myofibroblast-like cells, a key player in ECM production. Activated HSCs (myofibroblast-like cells) also produce TGF $\beta 1$, thus establishing both autocrine and paracrine loops for the abnormal accumulation of collagen in the liver.

A series of recent findings have raised the possibility that grafted MSCs may be able to reduce hepatic, cardiac, and peritoneal fibrosis through paracrine actions. Umbilical cordderived MSCs (UCMSCs) are known to reduce fibrosis of bleomycin-induced lung injury. Particularly, direct injection of exosomes isolated from UCMSCs reduces collagen accumulation in fibrotic mouse livers, suggesting that UCMSCs may secrete anti-fibrotic factors. However, the precise mechanisms of secretome-mediated tissue regeneration remain unclear. In this study, a single injection of the secretome obtained from UCMSCs significantly reduced liver fibrosis without cell grafting. Injection of the secretome into mice with liver fibrosis markedly decreased fibrillary collagen deposition and reduced the expression of fibrosis-related genes as well as activation of HSCs in the liver. Our in vitro study also showed that 


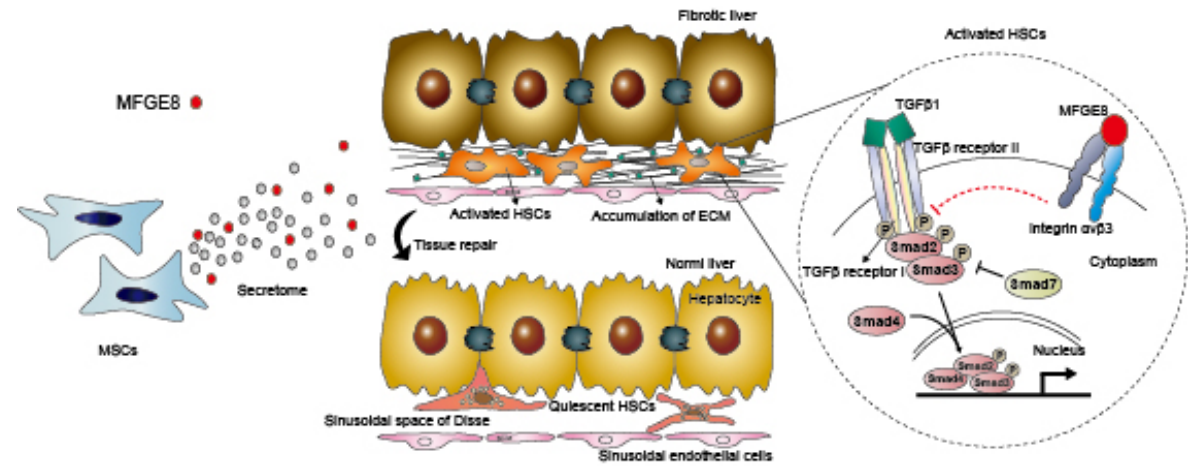

Fig. 1. Schematic model for MSC secretome-induced fibrotic regression in the liver. MSCs obtained from different tissues secrete many soluble factors, including MFGE8. MFGE8 binds to integrin $\alpha v \beta 3$ on hepatic stellate cells (HSCs) and downregulates the expression of TGFBR1. Perturbation of TGF $\beta$ signaling attenuates the activation of HSCs, a major source of ECM production, reducing the fibrogenic progression. secretomes diminished HSC activation without inducing apoptosis or senescence, and decreased the phosphorylation of Smad2 in the presence of TGF $\beta 1$. The results indicated that the primary action mechanism of secretome was achieved by inhibiting the TGF $\beta$ signaling pathway.

Using LC/MS and network analyses, we analyzed the UCMSC secretome and selected MFGE8 as a potential antifibrotic factor that contributes to the secretome-mediated reduction of liver fibrosis. MFGE8 is a soluble glycoprotein composed of an $\mathrm{N}$-terminal notch-like EGF domain with a highly conserved RGD motif and a C-terminal discoidin-like factor 5/8 factor domain. Our in vitro studies demonstrated that anti-MFGE8 neutralizing antibodies reduced the antagonistic activity of UCMSC secretome against HSC activation. In addition, recombinant MFGE8 protein downregulated the expression of TGFB type I receptor (TCFBR1) at the mRNA and protein levels, and decreased activation of HSC in the presence of TGF $\beta 1$. The MFGE8-mediated suppression of TCFBR 1 was inhibited by anti- $\alpha \mathrm{v} \beta 3$-integrin antibody, indicating that MFGE8 acts through $\alpha v \beta 3$ integrin. These results suggested a potential novel role for MFGE8 as a modulator of TGF $\beta$ signaling in HSCs. In addition, in vivo studies indicated that administration of the anti-MFGE8 antibody markedly and significantly reduced the anti-fibrogenic activity of the secretome. Moreover, when recombinant MFGE8 protein alone was administered to mice with liver fibrosis, the fibrotic area was significantly reduced. Histological studies using clinical samples demonstrated that the expression of MFGE8 was profoundly decreased in liver tissues of patients with cirrhosis, as compared to the normal liver. Therefore, MFGE8 may play an anti-fibrogenic role in endogenous regulation of liver fibrosis as well as in UCMSC secretome-mediated reduction of fibrosis.

In conclusion, these data collectively indicated that injection of MSC secretome significantly reduced fibrosis in the liver without cell transplantation. The anti-fibrotic effect of MSC secretome was mediated by inhibition of HSC-activation via regulation of TGF $\beta$ signaling (Fig. 1). In the MSC secretome, MFGE8 was identified as a novel key anti-fibrotic factor that disturb the TGF $\beta$ signaling.

\section{ACKNOWLEDGEMENTS}

This work was supported by a grant of the Korea Health Technology R\&D Project through the Korea Health Industry Development Institute (KHIDI), funded by the Ministry of Health and Welfare (HI14C1562) and the Bio and Medical Technology Development Program of the National Research Foundation (NRF), funded by the Ministry of Science, ICT \& Future Planning (MSIP), Republic of Korea (2012M3A9B40 28636). 